\title{
Mathematical Model of Drag Torque with Surface Tension in Single-Plate Wet Clutch
}

\author{
Zengxiong Peng ${ }^{*}$ (D) and Shihua Yuan
}

\begin{abstract}
Reduction of drag torque in disengaged wet clutches is essential for transmission research because it is one of the potentials of efficiency improvement. Aeration of oil film between two closely rotating plates promotes the decrease of drag torque at high speed region. The effects of surface tension and static contact angles during aeration are nonnegligible showed by test results. The traditional lubrication model does not adequately predict the experimental results with different surface tension and contact angles during aeration. Hence, in this present paper, contact angles between Aluminum and Teflon materials were firstly measured, and the drag torques under two different contact angles were examined experimentally. An improved lubrication model of drag torque based on Navier-Stokes equations at the gas-liquid interface was built. The lubrication boundary condition was modified to introduce the effects of surface tension and contact angle. The model shows that the effects at the beginning of aeration of oil film are significant. These effects almost occur at stationary plate due to low Reynolds number and Weber number. The model shows that an increase in the surface tension promotes aeration, but does not affect the peak drag torque. Increasing contact angle also promotes the aeration, and accelerates the decrease of drag torque. The larger contact angle is, the smaller the peak drag torque will be. A computational fluid dynamics (CFD) model based on volume of fluid (VOF) method was presented to validate the interface shape when aeration occurs. The model prediction has a good agreement with experimental observations for Aluminum plates and Teflon plates. The modified lubrication model of drag torque gives a convenient description of the effects of surface tension and contact angel, and lays down a frame to understand the beginning of aeration.
\end{abstract}

Keywords: Drag torque, Wet clutch, Surface tension, Contact angle

\section{Introduction}

The relative motion between the friction plates and separate plates in wet clutches causes a viscous shear force in tangential direction. This viscous force brings an inevitable torque named drag torque in open wet clutches. Reduction of drag torque in disengaged wet clutches is the focus of efficiency improvement because it is a countermeasure to reach this goal.

Traditional mode of drag torque based on Newton's law of viscosity can't predict the decrease when aeration occurs at a high rotating speed region. Several accurate mathematical models were presented in last decades. Hashimoto et al. [1] and Kato et al. [2] firstly

${ }^{*}$ Correspondence: pengzengx@bit.edu.cn

National Key Laboratory of Vehicular Transmission, Beijing Institute of Technology, Beijing 100081, China explained oil film aeration between the separate plate and the friction plate due to centrifugal force, which laid down a frame to model the drag torque accurately. Chinar et al. [3] developed a 2D lubrication model and a 3D CFD model considering grooved plates to evaluate the drag torque. The number, the depth of groove and the clearance between disks were investigated to understand their effects on the drag torque. The flow rate was very important in determining the pressure distribution along the plate and affects the drag torque. They also pointed out that aeration is the most important factor that reduces drag torque due to low viscosity of air. Kitabayashi et al. [4] made an analysis of various factors affecting drag torque in Multi-plate wet clutches. In their work, facing area, wave height, number of grooves, flow rate were discussed experimentally. Ryu et al. [5, 6] and Iqbal et al. [7] empirically 
determined the trends regarding geometric parameters, such as groove patterns, groove depths, flow rate and clearance between the friction plate and the separate plate. They also indicated the importance of wave height in reducing drag torque. In 2003, Yuan et al. [8, 9] simulated a three-dimensional, steady-state, twophase flow using the commercial CFD code FLUENT. Their results suggested that surface tension plays an important role in the incipience aeration. The work revealed that aeration after oil film shrinking is the main reason to result in the decrease of drag torque, because the viscosity of the oil is much less than that of the air.

CFD and experimental test are effective methods to study the drag torque of wet clutches when aeration occurs, but analytical lubrication model is beneficial to explain the mechanism of aeration. In the aspect of analytical lubrication model, $\mathrm{Hu}$ et al. [10] deduced the mathematical model for multi-plate wet clutches from Navier-Stokes equations. Their analysis revealed that oil flow acceleration in radial direction caused by centrifugal force is the key reason for aeration at the constant feeding flow rate. A circumferential degree was presented to calculate the liquid wet area after aeration. The model prediction agreed well with the test results for multi-plate wet clutches, but not for single-plate wet clutch. Against this problem, Yuan et al. [11, 12] investigated the flow pattern in the clearance of single wet clutch after aeration from visualization experiment and presented a flow pattern model different with Yuan et al. [9] when aeration occurs. Yet, the experimental results revealed that prediction model should be improved to explain the difference between model prediction and test data. They also pointed out that surface tension was one of the most possible countermeasures to be considered. Hu et al. [13] also proved that contact angle has a significant effect upon the drag torque.

In this work, the lubrication boundary condition in Yuan's [11] model is modified to introduce the effects of surface tension and contact angle. Then a modified model of drag torque is presented to prescribe contact line and to predict the interface shape when aeration occurs with varying surface tension coefficients and static contact angles. In order to validate the modified model, a small experimental rig was set up to test drag torque in single-plate wet clutch and to verify the effects of different contact angles. Section 2 discusses about the experimental results with different contact angles. Section 3 presents the derivation of the modified model considering surface tension and contact angle. Section 4 shows the validation of the model by numerical computation of VOF method and experiment results. Section 5 makes a conclusion.

\section{Experimental Apparatus and Result Discussion}

We set up an experimental rig to test the effects of surface tension and contact angle in Figure 1. As shown in Figure 2, the rotating plate connects to the motor shaft, and another plate is a stationary plate supported through a torque sensor. Several friction plates made up of different materials with different contact angle can be directly attached to the stationary plate. Oil pump supplies lubrication oil into the clearance through the flow sensor and the torque sensor. The torque sensor with hollow shaft is selected to avoid complicated seals for oil feeding. The torque sensor was pre-calibrated as range from $-5 \mathrm{~N} \mathrm{~m}$ to $5 \mathrm{~N} \mathrm{~m}$, and was capable of measuring accurately in small torque range by use of stain bridge. A $1 \mathrm{~kW}$ electrical heater installed in the tank allowed experiments at variable oil temperature.

The stationary plate was made from aluminum or aluminum coated with a 50-micron thick layer of Teflon. The static contact angle was measured on the aluminum and Teflon coated plate using a contact angle measurement device shown in Figure 3. The contact angle of aluminum is $10^{\circ} \pm 1^{\circ}$ and Teflon is $35^{\circ} \pm 1^{\circ}$. The geometric parameters of plates are summarized in Table 1, and the test conditions are shown in Table 2.

Figure 4 presents typical experimental results for drag torque $M$ as a function of rotating speed $\omega$. The data were tested with the aluminum plate and Teflon coated plate. Note that the drag torque increases linearly with the increasing rotating speed until there is a sharp drop, corresponding to the occurrence of aeration. It clearly reveals that increasing contact angle promotes aeration and leads the drag torque to dropping to nearly zero earlier. The torque ranges from 0 to $0.9 \mathrm{~N} \mathrm{~m}$ over a range of $0<\omega<2600$ $\mathrm{r} / \mathrm{min}$. The lubrication flow rate was fixed at $Q_{0}=3 \mathrm{~L} / \mathrm{min}$.

It is necessary to calculate Reynolds number and Weber number to distinguish the domination of vicious force, hydrodynamic force and surface tension force. The test

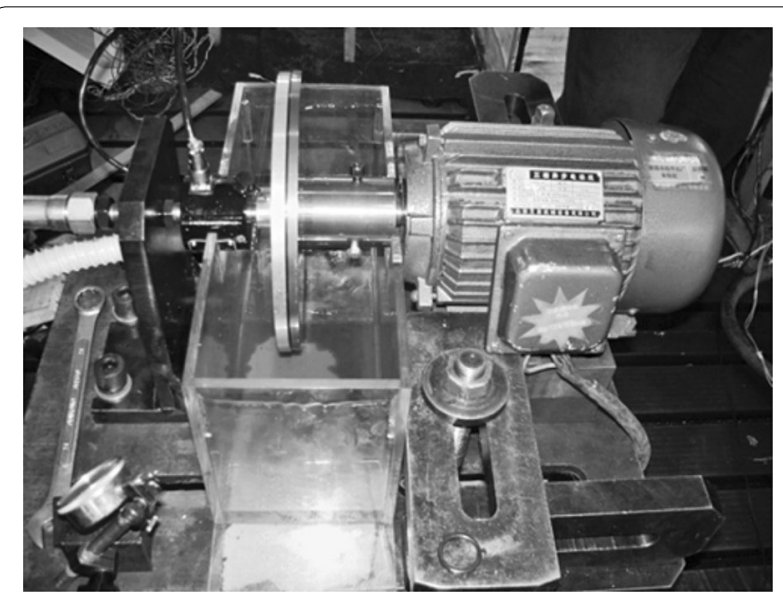

Figure 1 Experimental rig for single-plate wet clutch 


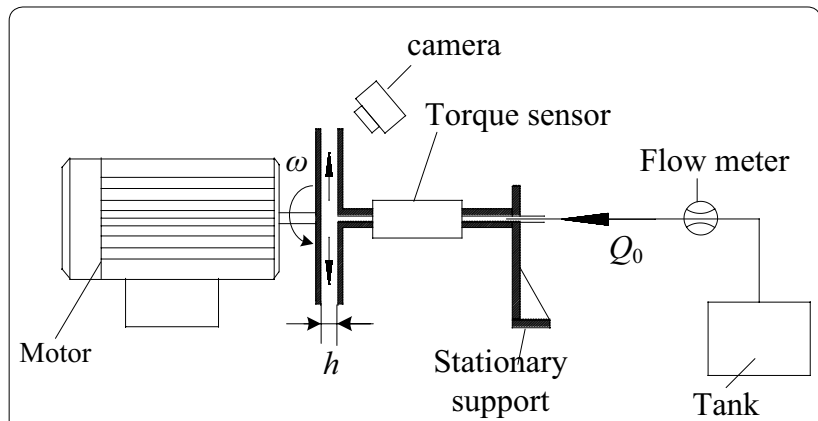

Figure 2 Block diagram of experimental rig for wet clutch

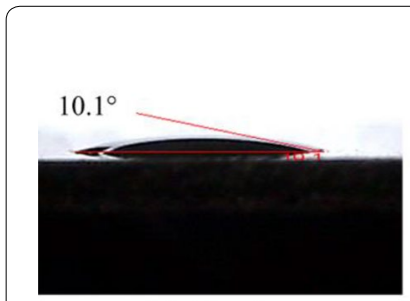

(a) Aluminum

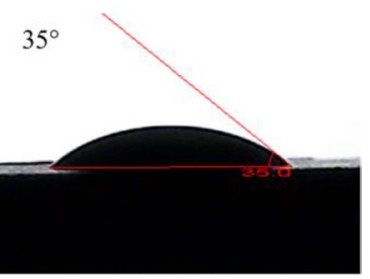

(b) Teflon
Figure 3 Contact angle test results

Table 1 Summary of plate geometric parameters

\begin{tabular}{lll}
\hline Outer radius $\boldsymbol{R}_{\mathbf{1}}(\mathbf{m m})$ & Inner radius $\boldsymbol{R}_{\mathbf{2}}(\mathbf{m m})$ & $\begin{array}{l}\text { Clutch clearance } \\
\boldsymbol{h}(\mathbf{m m})\end{array}$ \\
\hline 120 & 80 & 1 \\
\hline
\end{tabular}

Table 2 Summary of test conditions

\begin{tabular}{|c|c|c|c|c|}
\hline $\begin{array}{l}\text { Actual flow } \\
\text { rate } \\
Q_{0}(\mathrm{~L} / \mathrm{min})\end{array}$ & $\begin{array}{l}\text { Oil density } \\
\rho\left(\mathrm{kg} / \mathrm{m}^{3}\right)\end{array}$ & $\begin{array}{l}\text { Oil } \\
\text { viscosity } \\
\mu(\mathrm{Pa} \cdot \mathrm{s})\end{array}$ & $\begin{array}{l}\text { Temperature } \\
T\left({ }^{\circ} \mathrm{C}\right)\end{array}$ & $\begin{array}{l}\text { Surface } \\
\text { tension } \\
\text { coefficient } \\
\gamma(\mathrm{N} / \mathrm{m})\end{array}$ \\
\hline 3 & 872 & 0.066 & 30 & 0.035 \\
\hline
\end{tabular}

parameters above represent Reynolds number $R e=\rho \omega$ $R_{m} h / \mu$ in the range $0<\operatorname{Re}<360$, and Weber number $W e=\rho \omega^{2} R_{m}^{2} h / \sigma$ in the range $0<W e<18450$. Where $R_{m}$ is mean radius, and $\sigma$ is interfacial tension. Since Weber number is the ratio of hydrodynamic force to surface tension force, we have the conclusion that when $\operatorname{Re}>>1$, the contact angle effects can be neglected because of $W e>>1$ at rotating side. But it can't be neglected near stationary plate because Reynolds number and Weber number are nearly zero. This is the key reason that different contact angle affects drag torque near the stationary side shown in Figure 4 when aeration occurs.

Yuan et al. [11] pointed out that a difference still existed between their lubrication model prediction and test results, especially at the aeration range of $500 \mathrm{r} / \mathrm{min}<\omega<1000 \mathrm{r} /$ min. In the present work, we will introduce a contact line model to modify Yuan's model, so the effects of surface tension and contact line can be evaluated.

\section{Mathematical Model}

In the steady state, incompressible flow in the clearance of non-grooved disengaged wet clutch is shown in Figure 5. Brake mode is only considered here, so the separate plate is fixed.

Navier-Stokes equations can be written in cylindrical coordinates [14] as:

$$
\begin{aligned}
& \rho\left(v_{r} \frac{\partial v_{r}}{\partial x_{r}}-\frac{v_{\theta}^{2}}{r}\right)+\frac{\partial p}{\partial r}=\mu \frac{\partial^{2} v_{r}}{\partial z^{2}}, \\
& \rho\left(v_{r} \frac{\partial v_{\theta}}{\partial r}-\frac{v_{\theta} v_{r}}{r}\right)=\mu \frac{\partial^{2} v_{\theta}}{\partial z^{2}}, \\
& \frac{\partial p}{\partial z}=0
\end{aligned}
$$

where $\rho$ is the density of fluid, $\mu$ is the viscosity of fluid, $p$ is the pressure of fluid, $r$ is radius of the friction plate.

Shown as Figure 5, the boundary conditions for above equations can be written as:

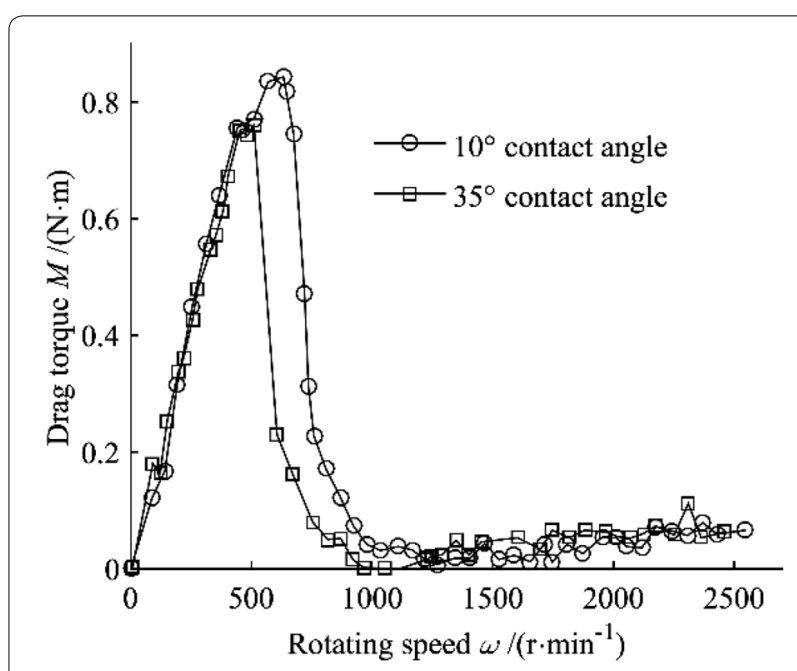

Figure 4 Drag torque results with different contact angles

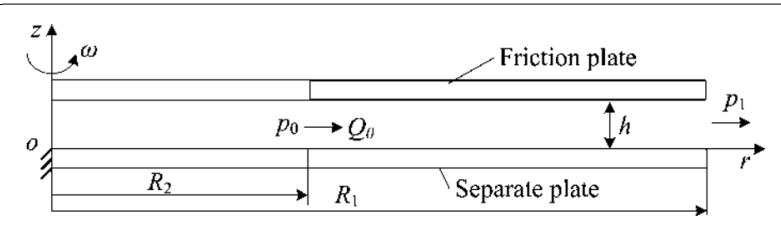

Figure 5 Schematic of disengaged wet clutch 


$$
\left\{\begin{array}{l}
v_{r}(r, 0)=0, v_{r}(r, h)=0, \\
v_{\theta}(r, 0)=0, v_{\theta}(r, h)=\omega r, \\
p_{2}=p_{0}, p_{1}=0
\end{array}\right.
$$

where $p_{1}$ is the exiting pressure at outer radius, $p_{2}$ is the flow pressure at inner radius, $p_{0}$ is the feeding pressure, $h$ is axial clearance.

Integrating Eq. (1) and considering the boundary conditions, we have the fluid radial speed distribution:

$$
\begin{aligned}
v_{r}=\frac{1}{2 \mu} & \frac{\mathrm{d} p}{\mathrm{~d} r} z(z-h)+\frac{\rho \omega^{2} r}{12 \mu h^{2}} z\left(h^{3}-z^{3}\right) \\
& +\frac{3 \rho Q^{2}}{20 \pi^{2} r^{3} \mu h^{6}} z\left(h^{5}-2 z^{5}+6 z^{4} h-5 z^{3} h^{2}\right),
\end{aligned}
$$

where $z$ is the axial distance of the gap, $Q$ is the ideal flow rate for single phase flow.

Ideal flow rate can be calculated through integration of radial speed as follows:

$$
\begin{aligned}
Q=\int_{0}^{2 \pi} \int_{0}^{h} V_{r} r \mathrm{~d} z \mathrm{~d} \theta=-\frac{\pi h^{3} r}{6 \mu} \frac{\mathrm{d} p}{\mathrm{~d} r}+\frac{\pi \rho \omega^{2} r^{2} h^{3}}{20 \mu} \\
+\frac{9 \rho Q^{2} h}{140 \pi \mu r^{2}} .
\end{aligned}
$$

Substituting Eq. (4) into Eq. (3) and rearranging, we have radial speed distribution:

$$
\begin{aligned}
v_{r}=- & \frac{3 Q}{\pi r h^{3}} z(z-h)+\frac{\rho \omega^{2} r}{60 \mu h^{2}} \\
& \times\left(9 z^{2} h^{2}-4 z h^{3}-5 z^{4}\right)+\frac{\rho Q^{2}}{140 \pi^{2} r^{3} \mu h^{6}} \\
& \times\left(27 h^{4} z^{2}-6 z h^{5}-42 z^{6}+126 z^{5} h-105 z^{4} h^{2}\right) .
\end{aligned}
$$

There is no surface tension factor in above model, and we attempt to use contact angle to reflect surface tension. The well-known Yong's equation describes the relationship between static contact angle and interfacial tensions on an ideal solid surface very well. In Young's equation [15]:

$$
\gamma_{s v}-\gamma_{s l}=\gamma_{l v} \cos \theta,
$$

where $\theta$ is contact angle, and $\gamma$ is surface tension coefficient, and the subscripts $s v, s l, l v$ refer to the solid-vapor, solid-liquid, liquid-vapor interfaces, respectively.

So we can use the change of contact angle to introduce the effect of surface tension. The experimental results reveal that contact angle and interface shape of the flow at the contact line affect aeration. If the static contact angle determines displacement of the flow between two disks, the dynamic contact angle determines the rate of the displacement. Wang et al. [16] provided a summary of empirical contact line models. There many authors developed contact line model based on Young's equation, and the commonly used contact line models are shown in Figure 6 .

The model on the left has a hysteresis region. When contact line begins to move, contact angle will change along with the moving velocity of the contact line. The simpler model is on the right without hysteresis region, which is appropriate for smooth surface [17].

At the liquid-vapor interfaces, surface tension alone governs the interface shape, and viscous stresses are negligible away from the wall [18]. Under the conditions of this present work, the flow is laminar and incompressible, and the air is passive. We can analyze this problem by using the contact line, which contains a contact angle. The velocity of contact line can be modeled by a simple expression:

$$
v_{c l}=\frac{1}{1000} \tanh ^{-1}\left(\theta-\theta_{s t}\right)\left(\theta_{\max }-\theta_{s t}\right)^{-1},
$$

where $\theta_{s t}$ is the static contact angle and $\theta_{\max }$ is the maximum contact angle in the appropriate quadrant.

In Eq. (7), we can see that if $\theta_{\max }$ is closer to $\theta_{s t}$ the velocity of contact line is larger. Both $\theta_{\max }$ and $\theta_{s t}$, are easy to get, and $\theta$ can be seen as a dynamic angle, we can get $\theta$ by using the equations as follows.

Based on Young's equation in wetting conditions,

$$
\sigma_{G S}=\sigma_{L S}^{0}+\sigma_{L G} \cos \theta_{D}+f+c \tau_{W} .
$$

In de-wetting conditions,

$$
\sigma_{G S}=\sigma_{L S}^{0}+\sigma_{L G} \cos \theta_{D}-f-c \tau_{W},
$$

where $\sigma_{L S}^{0}$ and $\sigma_{L S}$ are solid-liquid interfacial tensions under static and dynamic condition. $\sigma_{L G}$ is liquid-solid interfacial tension. $c$ is the proportional constant having dimension of length. $f$ is the hysteresis tension.

$$
\tau_{W}=-\frac{4 \mu U}{\lambda^{\prime}} \frac{\sin ^{2} \theta_{D}}{\sin \left(2 \theta_{D}\right)-2 \theta_{D}},
$$

$\tau_{W}$ is the stress singularity at the contact line. Where $\lambda^{\prime}$ is defined as the characteristic parameter of the contact

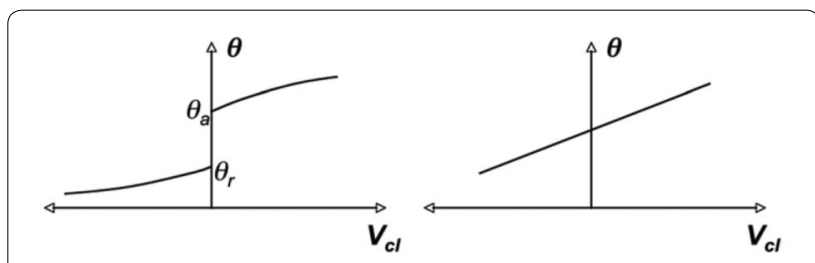

Figure 6 Commonly used contact line models 
line, and the hysteresis tensions $f$ in wetting and drying are approximately expressed as:

$$
\begin{aligned}
& f \approx f_{\max }=\sigma_{G S}-\sigma_{L S}-\sigma_{L G} \cos \theta_{a}, \\
& f \approx f_{\max }=-\sigma_{G S}+\sigma_{L S}+\sigma_{L G} \cos \theta_{a} .
\end{aligned}
$$

Combine Eqs. (8), (10), (11) and (9), (10), (12), we can have the dynamic contact angle.

For wetting:

$$
\cos \theta-\cos \theta_{a}=c \frac{4 \mu U}{\lambda \sigma_{L G}} \frac{\sin ^{2} \theta_{D}}{\sin \left(2 \theta_{D}\right)-2 \theta_{D}} .
$$

For dewetting:

$$
\cos \theta_{r}-\cos \theta=c \frac{4 \mu U}{\lambda \sigma_{L G}} \frac{\sin ^{2} \theta_{D}}{\sin \left(2 \theta_{D}\right)-2 \theta_{D}},
$$

where $\lambda=\lambda^{\prime} / c$ is a dimensionless characteristic parameter of contact line.

For systems with perfect equilibrium wetting $\theta_{a}=\theta_{r}=0$ and $\mu U / \sigma_{L G}<10^{-3}$. Eq. (13) and Eq. (14) are reduced to as follows:

$$
\theta_{D}^{3}=\frac{6 \mu U}{\sigma_{L G} \lambda} .
$$

Equation (15) is the well-known Hoffman-VoinovTanner law [16] at low capillary number, where $\lambda$ is a constant value determined by the experiment.

As the speed of contact line goes into the flow between two plates, a modified lubrication model is obtained by analyzing the problem in the contact line frame of reference where the contact line moves with the velocity of $V_{c l}$. We could think that the whole system moves as a speed when aeration occurs. A schematic of the model is shown in Figure 7. The speed of the top plate is $U-V_{c l}$ and that of the bottom plate is $-V_{c l}$. Thus the flow rate $Q^{\prime}$ will be rewritten as follows:

$$
Q^{\prime}=Q-v_{c l} h .
$$

Then the speed will be modified as follows:

$$
v_{r}^{\prime}=v_{r \max }-v_{c l} \text {. }
$$

According to Ref. [11], we can have:

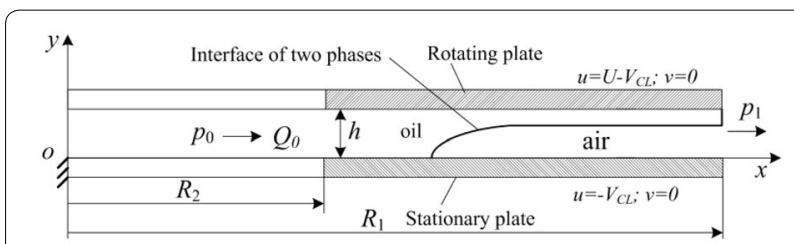

Figure 7 Schematic of interface of two phase

$$
\begin{aligned}
Q^{\prime}= & \frac{\frac{-6 \mu}{\pi h^{3}} \ln \frac{R_{1}}{R_{2}}}{0.039 h^{-2}\left(R_{1}^{-2}-R_{2}^{-2}\right)} \\
& +\frac{\sqrt{\left(\frac{6 \mu}{\pi h^{3}} \ln \frac{R_{1}}{R_{2}}\right)^{2}-\frac{81 \rho^{2} \omega^{2}}{700 \pi^{2} h^{2}}\left(R_{1}^{-2}-R_{2}^{-2}\right)\left(R_{2}^{2}-R_{1}^{2}\right)}}{0.039 h^{-2}\left(R_{1}^{-2}-R_{2}^{-2}\right)}-v_{c l} h .
\end{aligned}
$$

Then from Eq. (17), we derive the max value of the radial speed $v_{r \max }(r)$ from $\partial v_{r} / \partial z=0$, and define a new effective radius:

$$
r_{0}^{\prime}=\frac{Q_{0}}{2 \pi h v_{r}^{\prime}} .
$$

The shear stress on the rotating plates for singlephase flow is:

$$
\tau_{\theta z}=\left.\mu \frac{\partial v_{\theta}}{\partial z}\right|_{z=h}=\frac{\mu \omega r}{h} .
$$

The drag torque on each of the stationary plate then can be expressed as follows:

$$
M=2 \pi \int_{R_{1}}^{r_{0}^{\prime}} r \tau_{z \theta} r \mathrm{~d} r=\frac{\pi \mu \omega}{2 h}\left(r_{0}^{\prime 4}-R_{1}^{4}\right) .
$$

\section{Validation of the Model}

The flow in the clearance of wet clutches will change to two-phase flow after aeration. The numerical simulation of two-phase flows is a vast topic with a variety of applications ranging from basic fluid mechanics to engineering problems. The VOF method is first proposed by Hirt and Nichols [19] based on MAC method. It is widely used to solve the interactions owing to the capability of simulating the fragmentation and coalescence of fluid regions [20]. In the VOF method, the mixture is assumed to be uniform, and each phase has the same velocity and pressure in every control volume.

A two-phase model considering the surface tension was built in FLUENT software. The model was presented in 2D instead of $3 \mathrm{D}$ due to the axisymmetric geometric structure. Thereby, the mesh cells are diminished, and the calculation efficiency and precision are greatly improved. The number of mesh nodes in radial and axial directions is 200 and 60, respectively. The model parameters is the same as Table 1 and Table 2 .

The inlet boundary was specified as "mass flow inlet", and the outlet boundary was specified as "pressure outlet" (atmospheric pressure). Rotating plate was specified as a rotating wall, while stationary plate was specified as a stationary wall. The surface tension was turned on. The volume fraction of oil in the whole region was initialized as 0 , and then it will vary automatically based on the governing equations. 
Figure 8 illustrates the interface sharp of two-phase flow when aeration occurs. Without aeration, the flow is considered fully flooded. But when the speed is up to a critical value, the gas can penetrate from the outer radii of the plates to partially aeration the gap flow. The aeration usually forms on the stationary plate. Comparing with centrifugal force at rotating plate, the force of surface tension can be neglected. Aeration at the inner radii of the wet clutch plates depends on the formation of a free surface and contact line on the stationary plate [21]. This suggests that interfacial properties of the oil and plate surfaces will be an important consideration.

Figure 9 presents three drag torque curves versus rotating speed with different surface tension coefficient, which were computed by the modified model. As the speed increases, the beginning drag torque increases nearly linearly. However, the drag torque drops to near zero because of aeration when the speed rises up to a critical value, as shown in Figure 9. An increase in the surface tension promotes aeration and leads the drag torque drop to near zero more quickly.

The test results in Figure 10 were attained by varying contact angle $\theta$ while keeping the surface tension fixed. A higher contact angle means an oleophobic behavior of the stationary plate, which promotes the aeration and reduces the drag torque.

To validate the modified model presenting above, two different stationary plates with Aluminum and Teflon material were tested. The comparisons of model prediction with the clutch experiment are shown in Figure 11. The experimental results illustrate the influence of different contact angles with Aluminum plate and the Teflon coated plate. The contact angle can be used for evaluating the effects of the surface tension indirectly. A larger contact angle indicated a lesser affinity for oil. As a result, the increasing contact angle promotes aeration.

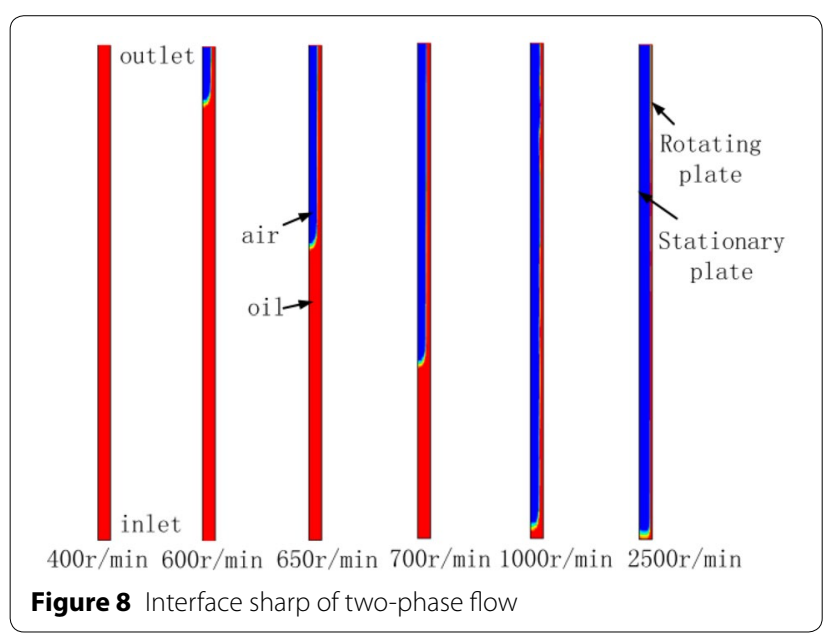

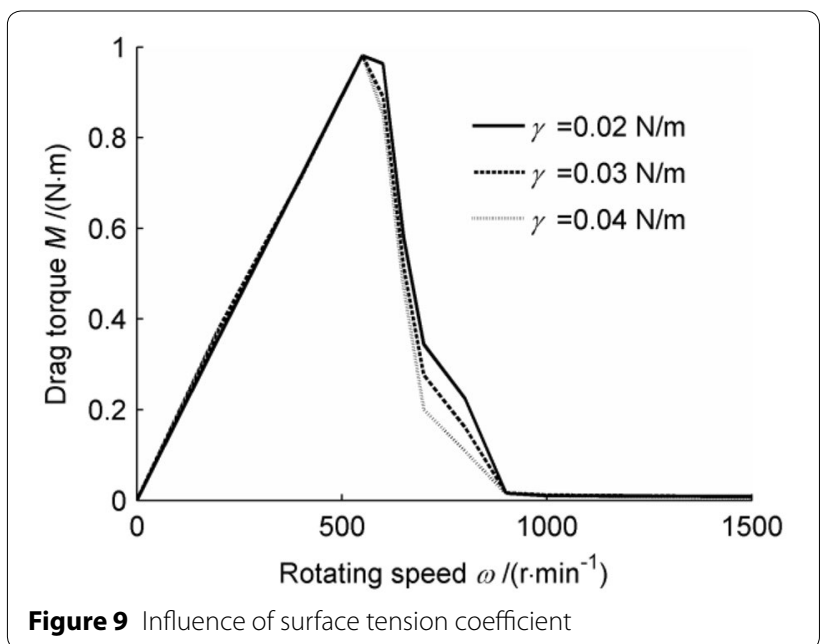

\section{Conclusions}

(1) A gas layer is formed on the stationary plate because the centrifugal force of oil near the stationary plate is low compared to that near the rotating plate. The effects of surface tension and contact angle on occurrence of aeration at the stationary side of the wet clutch are significant. A modified lubrication model considering surface tension and contact angle is presented. The model including the dynamic contact line modifies the speed distribution of flow and introduces the influence of the surface tension and contact angle.

(2) Larger surface tension coefficient and larger contact angle promote the aeration in favor of reducing drag torque. Improving the oleophobic behavior

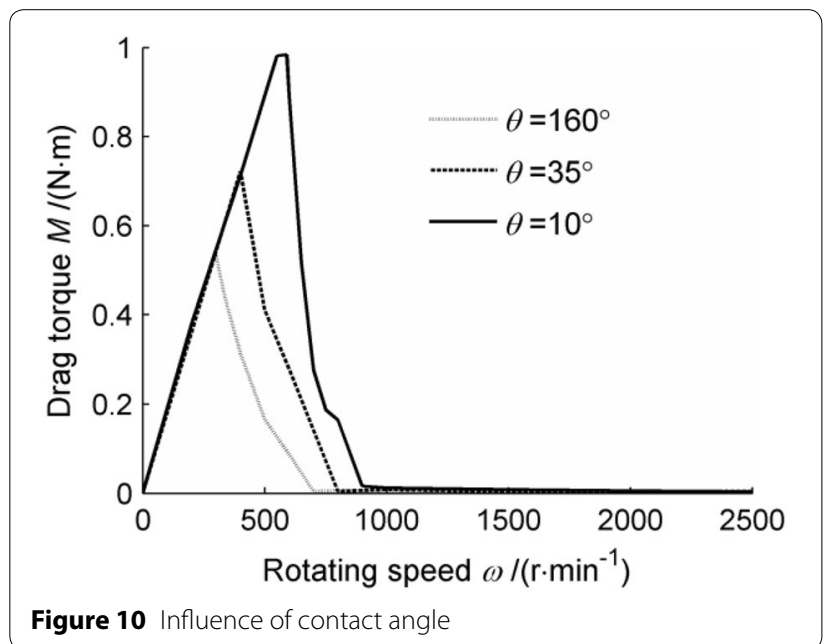




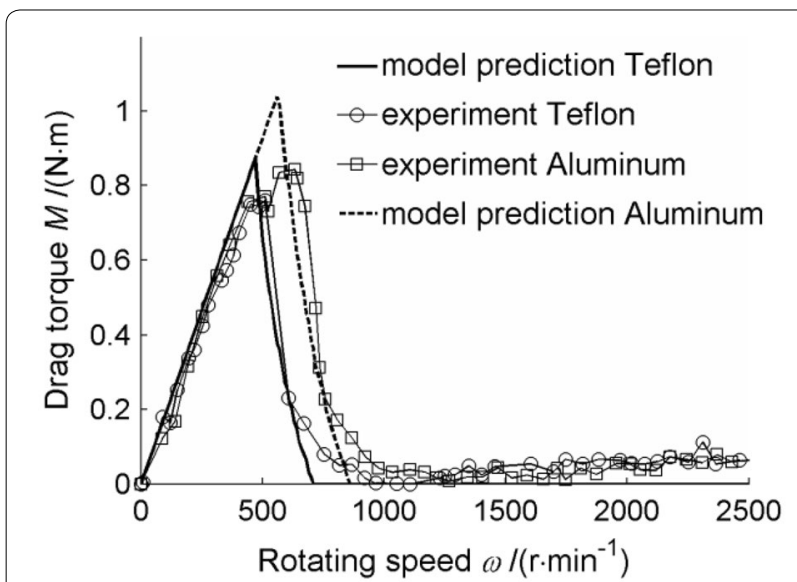

Figure 11 Comparisons of model prediction and test results

of the stationary plate is one of measures to reduce drag torque, and the lubrication oil with low surface tension coefficient is also helpful.

(3) The modified model is validated by CFD analysis and experimental results. This model is successfully used for predicting the curve of drag torque with different contact angle and laying down a frame to understand the process of aeration.

\section{Authors' Contributions}

SY was in charge of the whole trial; ZP wrote the manuscript; ZP assisted with sampling and laboratory analyses. Both authors read and approved the final manuscript.

\section{Authors' Information}

Zengxiong Peng, born in 1979, is a PhD candidate at School of Mechanical Engineering, Beijing Institute of Technology, China. He received his master degree from School of Mechanical Engineering, Beijing Institute of Technology, China, in 2006. His research interests include vehicle hydraulic transmission, hydraulic control, etc.

Shihua Yuan, born in 1958, is currently a professor at Beijing Institute of Technology, China. He received his PhD degree from Beijing Institute of Technology, China, in 1999. His research interests include the technology of vehicular transmission and hydraulic transmission.

\section{Competing Interests}

The authors declare that they have no competing interests.

\section{Funding}

Supported by National Natural Science Foundation of China (Grant No. 51305032).

Received: 13 October 2017 Accepted: 15 March 2019

Published online: 25 March 2019

\section{References}

[1] H Hashimoto, S Wada, Y Murayama. The performance of a turbulent-lubricated sliding bearing subject to centrifugal effect. Trans. Jpn. Soc. Mech. Eng. Ser. C, 1984, 49(446): 1753-1761. (in Japanese)

[2] Y Kato, T Murasugi, H Hirano, et al. Fuel economy improvement through tribological analysis of the wet clutches and brakes of an automatic transmission. Society of Automotive Engineers of Japan, 1993, 16(12): 57-60.

[3] C R Chinar, Jinhyun Cho, W W Schultz, et al. Modeling and parametric study of torque in open clutch plates. Journal of Tribology, 2006, 128(2): 422-430.

[4] H Kitabayashi, Yuli Chen, $\mathrm{H}$ Hiraki. Analysis of the various factors affecting drag torque in multiple-plate wet clutches. JSAE/SAE International Spring Fuels \& Lubricants Meeting, Yokahama, Japan, May 19-22, 2003: 1-6.

[5] J S Ryu, I H Sung. Effect of angle and density of grooves between friction plate segments on drag torque in wet clutch of automatic transmission. J. Korean Soc. Tribol. Lubr. Eng., 2014, 30(2): 71-76. (in Korean)

[6] Ryu J S, I H Sung. Effects of friction plate area and clearance on the drag torque in a wet clutch for an automatic transmission. J. Korean Soc. Tribol. Lubr. Eng., 2014, 30(6): 337-342. (in Korean)

[7] I S qbal, F Al-bender, B Pluymers, et al. Experimental characterization of drag torque in open multi-disks wet clutches. SAE International Journal of Fuels and Lubricants, 2013, 6(3): 894-906.

[8] Yiqing Yuan, Pradeep, Dong Yu. CFD simulation of the flows within disengaged wet clutch of an automatic transmission. SAE World Congress, Michigan, USA, March 3-6, 2003: 1-6.

[9] Yiqing Yuan, Eysion Liu, H James. An improved hydrodynamic model for open wet transmission clutches. Journal of Fluids Engineering, 2007, 129(3): 333-337

[10] Jibin Hu, Zengxiong Peng, Shihua Yu. Drag torque prediction model for the wet clutches. Chinese Journal of Mechanical Engineering, 2009, 22(2): 238-243.

[11] Shihua Yuan, Zengixong Peng, Chongbo Jing. Experimental research and mathematical model of drag torque in single-plate wet clutch. Chinese Journal of Mechanical Engineering, 2011, 24(1): 91-97.

[12] Shihua Yuan, Kai Guo, Jibin Hu, et al. Study on aeration for disengaged wet clutches using a two-phase flow model. Journal of Fluids EngineeringTransactions of the ASME, 2010, 132(11): 111304-1-111304-6.

[13] Jibin Hu, Zengxiong Peng, Chao Wei. Experimental research on drag torque for single-plate wet clutch. Journal of Tribology-Transactions of the ASME, 2012, 134(1): 014502-1-014502-6.

[14] Jinshi Chang. A calculation formula for carrying-capacity of annular hydrostatic step-bearing taking account of oil-flow inertia. Journal of Mechanical Engineering, 1982, 18(1): 62-68. (in Chinese)

[15] J G Robert. Contact angle, wetting, and adhesion: a critical review. Journal of Adhesion Science and Technology, 1992, 6(12): 1269-1302.

[16] Xiaodong Wang, Xiaofeng Peng, Duzhong Lee. Dynamic wetting and stress singularity on contact line. Science in China, 2003, 46(4): 407-415.

[17] Y D Shikhmurzaev. The moving contact line on a smooth solid surface. International Journal of Multiphase Flow, 1993, 19(4): 589-610.

[18] V Gauri, KW Koelling. The motion of long bubbles through viscoelastic fluids in capillary tubes. Rheologica Acta, 1999, 38(5): 458-470.

[19] CW Hirt, B D Nichols. Volume of fluid (VOF) method for the dynamic of free boundaries. Journal of Computational Physics, 1981, 39(1): 201-225.

[20] Yu-ting Ma, Zhi-guo Pei, Zhong-xiang Chen. Multi-field analysis and experimental verification on piezoelectric valve-less pumps actuated by centrifugal force. Chinese Journal of Mechanical Engineering, 2017, 30(4): 1032-1043.

[21] R A Chinar, W S William, L C Steven. Aeration in lubrication with application to drag torque reduction. Journal of Tribology, 2011, 133(3): 031701-1-031701-7. 